\title{
Surface Plasmon Microscopy of Two Crystalline Domains in a Lipid Monolayer
}

\author{
R. P. H. Kooyman* \\ Department of Applied Physics, Twente University, P.O. Box 217, \\ 7500 AE Enschede, The Netherlands \\ U. J. Krull \\ Department of Chemistry, University of Toronto at Erindale, Mississauga, \\ Ontario L5L 1C6, Canada
}

Received September 10, 1990. In Final Form: December 21, 1990

\begin{abstract}
Surface plasmon microscopy is applied to monolayers of dimyristoylphosphatidylethanolamine, dipcast at high lateral pressure $(35 \mathrm{mN} / \mathrm{m})$ on a solid substrate. The vertical resolution of better than $1 \mathrm{~nm}$ and an in-plane resolution of $\sim 10 \mu \mathrm{m}$ allow for the detection of two separate solid domains. Assuming a fixed value for the lipid refractive index, the tilt angles of the lipid aliphatic chains were determined to be $0^{\circ}$ and $\sim 35^{\circ}$, respectively.
\end{abstract}

\section{Introduction}

The study of the organization of amphiphilic molecules in a monolayer deposited on a water or solid substrate has been an active research topic for a number of years. ${ }^{1,2}$ It is well-known that these molecules may exhibit solid (ordered) or fluid (disordered) behavior; moreover, depending on the applied surface pressure, these phases can coexist resulting in a surface domain structure as was suggested by inflections in pressure-area curves and by fluorescence microscopy (FM) . $^{3,4}$

Up to now the technique of FM has been one of the very few that could be applied to directly view domain structures. Its success is based on the fact that a properly chosen fluorescent probe, incorporated in the monolayer, has a supposedly very limited solubility in the solid phase. Thus, under the fluorescence microscope, solid domains in a fluid environment appear as dark patches in a bright background.

Apart from the facts that the partition coefficients between domains for common dyes are not well-known and that the presence of the dye might perturb the domain structure, an important disadvantage of this method is that it is very difficult to obtain quantitative information on the nature of these domains. Now, on one hand, ellipsometric experiments ${ }^{5,6}$ indicate that these monolayers change their layer thickness and refractive index, dependent on the applied lateral pressure, which is a consequence of the changed packing density and hydrocarbon tail conformation. On the other hand, Rothenhäuser and Knoll ${ }^{7}$ have recently introduced surface plasmon microscopy (SPM) to image surface structures with small differences in refractive index and/or layer thickness. A vertical resolution of better than $1 \mathrm{~nm}$ was claimed, for a lateral resolution of $\sim 10 \mu \mathrm{m}$. Thus SPM,

* Author to whom correspondence should be sent.

(1) Andelman, D.; Brochard, F.; Joanny, J. J. Chem. Phys. 1987, 86, 3673.

(2) Gaines, G. Insoluble Monolayers at Liquid-Gas Interfaces; Interscience Publishers: New York, 1966.

(3) Tscharner, V.; McConnell, H. M. Biophys. J. 1981, 36, 409.

(4) Lösche, M.; Sackmann, E.; Möhwald, H. Ber. Bunsen-Ges. Phys. Chem. 1983, 87, 848 .

(5) den Engelsen, D.; de Koning, B. J. J. Chem. Soc., Faraday Trans. $11974,70,1603$.

(6) Huang, W. T.; Levitt, D. G. Biophys. J. 1977, 17, 111.

(7) Rothenhäusler, B.; Knoll, W. Nature 1988, 332, 615. applied to a monolayer domain structure, is expected to reveal the separate domains, exploiting the intrinsic properties of the system under study.

The feasibility of this approach has been demonstrated very recently by Knoll's group. ${ }^{8}$ We have set up a similar SPM system which enabled us to describe a new twosolid-phase equilibrium within a lipid monolayer.

\section{Surface Plasmon Microscopy}

As SPM is a relatively unfamiliar technique in lipid monolayer research, it seems appropriate to give a short description here; for details concerning the theory of surface plasmon resonance and the mechanism of image formation we refer to Raether's review ${ }^{9}$ and to recent SPM papers. 7,8

Consider the following arrangement: A prism (refractive index $\left.n_{1}\right)$ is coated with a thin $(\sim 50 \mathrm{~nm})$ metal overlayer $\left(n_{\mathrm{m}}\right.$, complex $)$, which is in contact with a medium of refractive index $n_{2}<n_{1}$. p-Polarized light with wavelength $\lambda$, falling through the prism, reflects at the metal-prism interface; the intensity of the reflected light is measured. At some angle, $\theta_{0}$, a sharp minimum is found in the reflectance. At this angle a collective motion of the conduction electrons (a surface plasmon) with coherence length $L_{\mathrm{c}}$ of the order of $10 \mu \mathrm{m}$ in the metal is excited. ${ }^{8}$

From Maxwell's equations it can be calculated that for this situation a strong evanescent electric field is built up at the interface $n_{\mathrm{m}} / n_{2}$. The amplitude of this field decays into medium $n_{2}$ with a characteristic distance of the order of $\lambda / 2$. If an extra layer is placed between metal and the medium, then it is found that, owing to the strong localization of the field at the interface, the resonance angle is strongly dependent on the thickness and refractive index of this layer. We have calculated ${ }^{10}$ that a thickness resolution of ca $0.05 \mathrm{~nm}$ can be obtained for an experimental $\theta_{0}$ resolution of $0.01^{\circ}$.

Thus, the surface plasmon resonance condition will be determined by the spatial average over $L_{c}$ of the refractive

(8) (a) Hickel, W.; Knoll, W. Thin Solid Films 1990, 187, 349. (b) Hickel, W.; Kamp, D.; Knoll, W. Nature 1989, 339, 186. (c) Hickel, W. Knoll, W. J. Appl. Phys. 1990, 67, 3572.

(9) Raether, H. Surface Plasmons on Smooth and Rough Surfaces and on Gratings; Springer: Berlin, 1988.

(10) Kooyman, R. P. H.; Kolkman, H.; van Gent, J.; Greve, J. Anal. Chim. Acta 1988, 213, 35. 


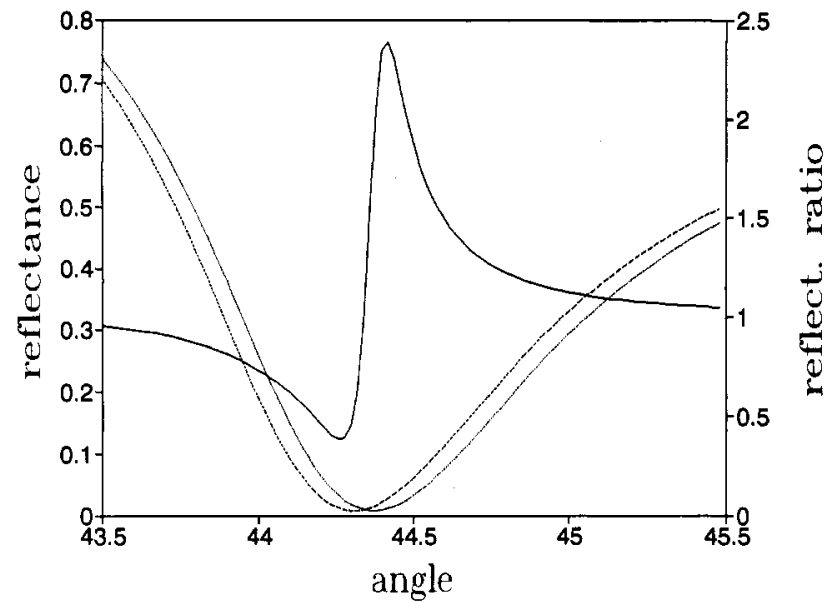

Figure 1. Calculated SPR reflection curves for $\lambda=633 \mathrm{~nm}, 49$ $\mathrm{nm} \mathrm{Au}, 2 \mathrm{~nm} \mathrm{SiO} \mathrm{Si}_{2}$, and an overlayer with $n=1.5$ and thickness $t$ : dashed, $t=2.0 \mathrm{~nm}$; dotted, $t=2.5 \mathrm{~nm}$; solid line, reflectance ratio.

index profile near the metal surface; differences in this profile over distances $>\sim L_{\mathrm{c}}$ will be detected as a change in the resonance angle. In practice, if light is incident on the prism with a fixed angle, this results in nearly complete light absorption at only those positions where the plasmon is excited. By magnification of the illuminated spot, the resonant positions are discernible as dark structures in a bright background. Because the resonance curve can be made as small as $0.2^{\circ}$ by proper choice of wavelength and materials, the intensity ratio (the contrast) between two illuminated similar structures can be very high. This is illustrated in Figure 1 where Fresnel's equations (e.g. see ref 9) were used to depict the contrast as a function of the angle of incidence for a gold layer of $49 \mathrm{~nm}$ on which layers of identical refractive index with thickness of 2.0 and $2.5 \mathrm{~nm}$ are deposited, respectively. It is seen that the contrast can be as high as 2.5 , which is very easy discernible. Furthermore, it is obvious from Figure 1 that for this particular two-component structure the contrast can be inverted. In practice, this latter feature proves to be very useful in discriminating SPM images from spurious signals.

\section{Materials and Methods}

Dimyristoylphosphatidylethanolamine (DMPE) was chosen as the monolayer-forming compound, because it is $\mathrm{known}^{11}$ that it forms relatively large domain structures.

Monolayers of DMPE, obtained from Sigma were prepared as follows: About $100 \mu \mathrm{L}$ of a $1 \mathrm{mg} / \mathrm{mL}$ solution of DMPE in $3: 1$ $(\mathrm{v} / \mathrm{v})$ chloroform/methanol was spread in $1-\mu \mathrm{L}$ increments onto the subphase $\left(1 \mathrm{mM} \mathrm{CdCl}{ }_{2}\right.$ in deionized water, initial specific resistivity $>15 \mathrm{M} \Omega \mathrm{cm}, \mathrm{pH} \sim 7.5$ in a Langmuir trough (Mgw. Lauda), equipped with a Wilhelmy plate for surface pressure transduction. After evaporation of the solvent, the lipid layer was compressed to near the collapse pressure and expanded. Subsequently, the monolayer was very slowly compressed to the desired lateral pressure.

Lipid monolayers with or without $1 \mathrm{~mol} \%$ of added fluorescent dye nitrobenzoxadiazole dipalmitoylphosphatidylethanolamine (NBD-DPPE) were transferred to microscope slides (Fisher) that were prepared in the following way:

After a cleaning procedure, consisting of soaking in a detergent solution in an ultrasonic bath for $1 \mathrm{~h}$ followed by extensive washing in water, $\sim 50 \mathrm{~nm}$ of gold (purity $>0.9999$ ) was evaporated in a vacuum chamber, evacuated to $0.5 \mu \mathrm{Torr}$. The cleaning procedure was essential in order to provide good adhesion between the gold layer and the glass slide. Gold was chosen as the metal to support surface plasmons, because this material exhibits a relatively small

(11) Heckl, W. M.; Lösche, M.; Scheer, M.; Möhwald, H. Biochim. Biophys. Acta 1985, 810, 73 .

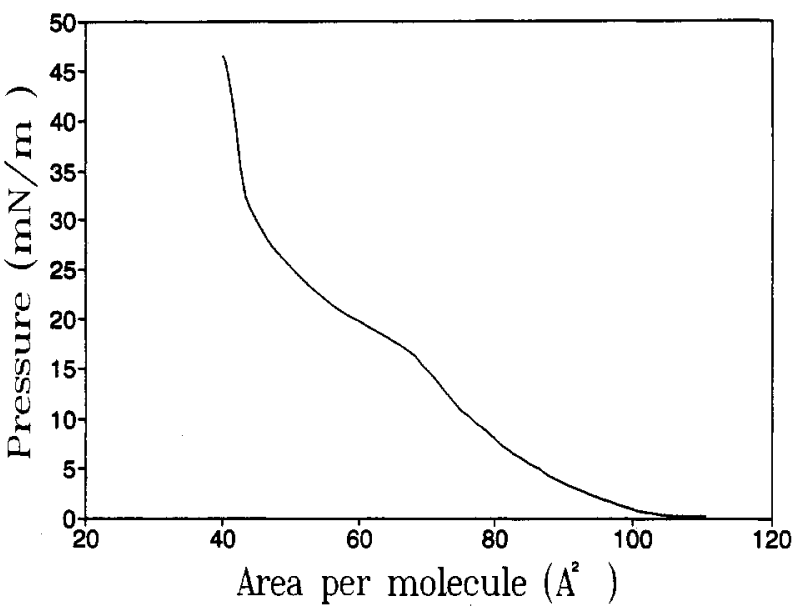

Figure 2. Pressure-area curve of DMPE on $1 \mathrm{mM} \mathrm{CdCl} 2-$ aqueous subphase, temp $22^{\circ} \mathrm{C}$.

SPR line width $\left(\sim 0.5^{\circ}\right)$ corresponding to a reasonable propagation length; $L_{\mathrm{c}} \sim 10 \mu \mathrm{m}$. Furthermore, gold is relatively inert with respect to chemical reactivity. As we found that gold has rather unpredictable properties with respect to its surface hydrophilicity (after the deposition the contact angle could vary between $10^{\circ}$ and $90^{\circ}$ for different samples), it was necessary to coat the gold layer with an overlayer that was stable in this respect. A sputtered thin layer of $\mathrm{SiO}_{2}$ (thickness 1-10 nm) on top of the gold proved to give a satisfactory hydrophilic surface for dipcasting purposes. The $\mathrm{SiO}_{2}$ layer thickness was determined by SPR and subsequent fitting of the experimental data to Fresnel's equations (e.g., see ref 9 ).

Dipcasting was done by partial vertical immersion and subsequent lifting of a metalized and overcoated glass slide at a rate of $6 \mathrm{~mm} / \mathrm{min}$; in this way both the bare substrate and the monolayer on the same slide could be studied. As expected, monolayer transfer occurred in the upward stroke. Only those samples with a transfer ratio of $1.00 \pm 0.14$ were further studied with the SPM instrumentation.

A SPM was built, essentially in the Kretschmann configuration. ${ }^{9}$ A $2-\mathrm{mW}$ unpolarized $\mathrm{HeNe}$ laser $(\lambda=633 \mathrm{~nm})$ was employed as a light source. The polarization and intensity of the incident beam could be adjusted by a pair of polaroid sheets.

The microscope slides were optically coupled to the prism by using a matching immersion oil $(n=1.51)$. A $f=6 \mathrm{~cm}$ lens was placed in the path of the exiting light beam. The BK7 $90^{\circ}$ prism (Melles Griot) was placed upon a XYZ translation stage. The angle of incidence could be adjusted by a dc/ac powered vibrating mirror. ${ }^{12}$ The illuminated spot was imaged onto a simple videocamera; the image could be fed into a microcomputer by using a video frame grabber. In order to analyze the SPM data it was assumed that layers deposited on the glass substrate could be taken as perfectly smooth and homogeneous over a distance much larger than the used wavelength. In this approximation the layer system can be described in a fairly straightforward way by using Fresnel's reflection equations (see, e.g., ref 9 ).

Finally, it should be mentioned that all angular values, quoted in the following, are measured within the prism.

\section{Results and Discussion}

In Figure 2 the compression curve of DMPE is depicted. The general appearance and the collapse pressure of $\sim 50$ $\mathrm{mN} / \mathrm{m}$ are consistent with previous results. ${ }^{11}$ In the region around $0.6 \mathrm{~nm}^{2} /$ molecule, fluid and solid phases coexist, and it is here where Hickel and $\mathrm{Knoll}^{8}$ found a close correspondence between the SPM and the FM images. We found a similar behavior; rather than duplicating their results here, we will concentrate on the regime where the lipid is dipcast while mainly in its solid state, i.e. around a pressure of $\sim 35 \mathrm{mN} / \mathrm{m}$.

(12) Kooyman, R. P. H.; Lenferink, A.; Eenink, R. G.; Greve, J. Anal Chem. 1991, 63, 83. 

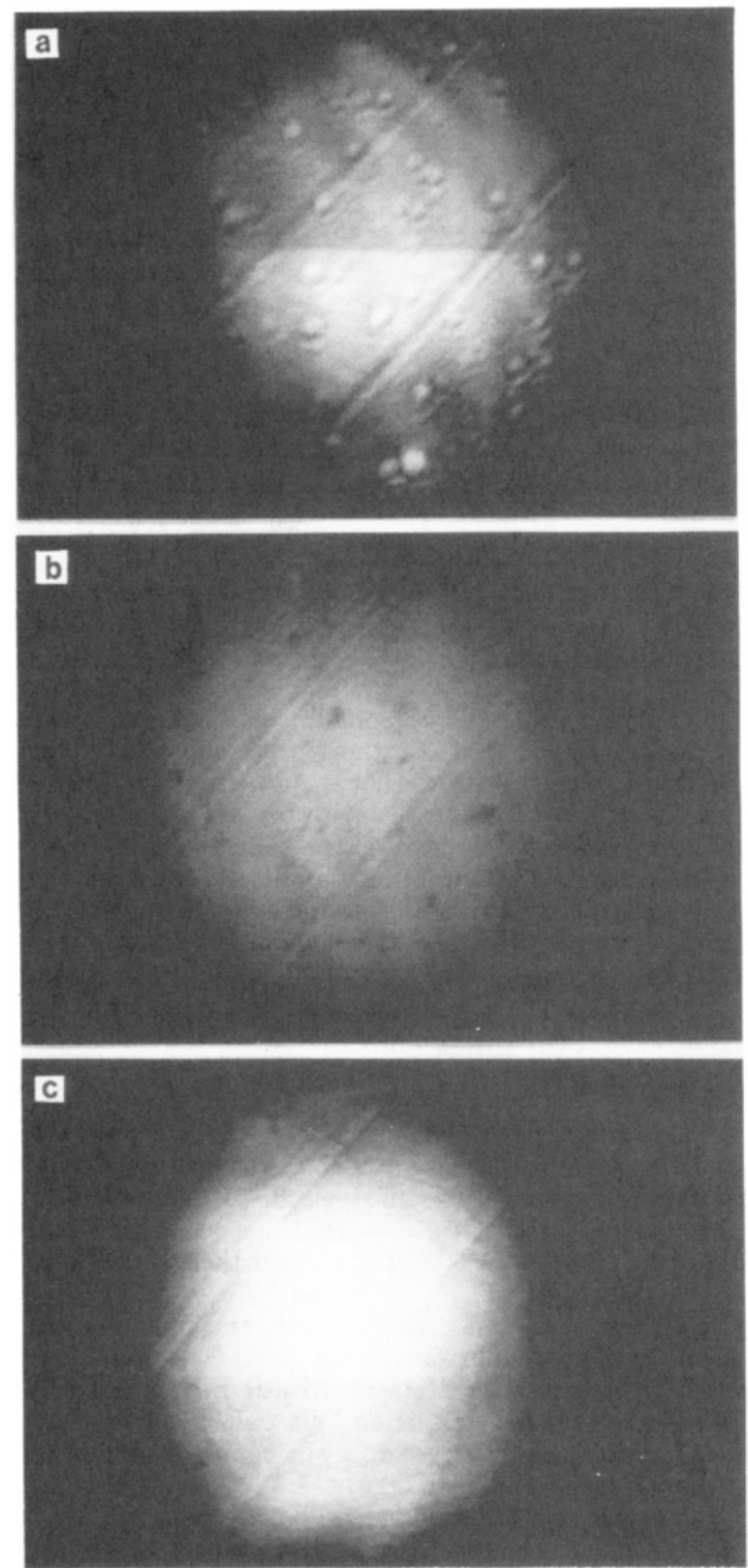

Figure 3. Experimental SPM images of DMPE monolayer on solid substrate (cf. text). No contrast enhancement was applied. Photographs taken from the digitized images. Area shown $\sim 250$ $\times 200 \mu \mathrm{m}^{2}$. The lines are artifacts originating from scratches in the glass surface: (a) $\varphi=44.5$; (b) $\varphi=44.7$; (c) $\varphi=45.1$.

SPM images are shown of a monolayer prepared at 35 $\mathrm{mN} / \mathrm{m}$ in Figure 3. It can be seen in the sequence of images, where the total change of angle of incidence is $\sim 0.6^{\circ}$, that bright patches with diameter $\sim 25 \mu \mathrm{m}$ (a) turn into dark ones (b), whereas in part c the image is essentially featureless.

The findings that the image contrast can be reversed within $0.5^{\circ}$ and that above a certain angle the contrast is absent point strongly to the fact that surface plasmons are excited in separate lipid domains; the results in Figure 3 appear to be an experimental demonstration of the simulation shown in Figure 1. The following checks substantiate this interpretation:

(1) Changing the incident beam to s-polarized light at the resonance angle resulted in the complete disappearance of the contrast; the same holds for illumination with p-polarized light, at ca. $1^{\circ}$ from resonance.

(2) Excitation of a SP at the bare $\mathrm{SiO}_{2} / \mathrm{Au}$ surface resulted in a homogeneously illuminated image without structure. Apparently, the intrinsic roughness of a sputtered $\mathrm{SiO}_{2}$ layer has lateral dimensions much smaller than the SPR propagation length.

(3) Excitation of a SP at a $\mathrm{SiO}_{2} / \mathrm{Au}$ surface, dipcast in the neat subphase, resulted in a nearly featureless image, similar to the bare $\mathrm{SiO}_{2} / \mathrm{Au}$ surface.

In addition, the presence and quality of monolayers in terms of regularity and domain formation were confirmed by FM observations of freely floating membranes as well as monolayers that were cast onto glass slides without underlying metal coating.

The images, depicted in Figure 3, can therefore be attributed with some confidence to the excitation of SP's in lipid regions with different properties. (The fact that all images show an approximately equal gray level comes from the circumstance that we used an inexpensive videocamera with automatic gain control, in which contrasts tend to be diminished.)

The resonance angles for a BK7-slide/49 nm Au/1 nm $\mathrm{SiO}_{2}$ substrate we found to be

$$
\varphi=44.25 \pm 0.03 \text { substrate }
$$

$$
\begin{gathered}
\varphi=44.52 \pm 0.03 \text { lipid "background" domain I } \\
\varphi=44.65 \pm 0.05 \text { lipid patch domain II }
\end{gathered}
$$

Fresnel's equations were used to determine the corresponding layer thicknesses, with the gold optical constants taken from the literature. ${ }^{13}$ and $n_{\mathrm{SiO}_{2}}=1.46$. We assumed the lipid refractive index to be $n_{1}=1.50-1.54^{5,6,14}$ and ignored the circumstance that organized lipid layers are birefringent.

The following values are found for the lipid layer thicknesses:

$$
\begin{aligned}
& t=2.3 \pm 0.3 \mathrm{~nm} \quad\left(n_{1}=1.50\right) \text { domain I } \\
& t=3.3 \pm 0.4 \mathrm{~nm} \quad\left(n_{1}=1.52\right) \quad \text { domain II }
\end{aligned}
$$

The larger value for $n_{1}$ that we used for domain II as compared to that used for domain I reflects the anticipated increase in density of these small domains. However, the quoted values of the thickness are not very sensitive to the choice of $n_{1}$ : changing the value of $n_{1}$ in domain II with \pm 0.02 results in a calculated change of layer thickness of $\mp 0.1 \mathrm{~nm}$. Note also that the calculated thickness values virtually rule out the presence of multilayers.

For a further interpretation of the SPM results it is appropriate to discuss the FM image of a monolayer cast on a slide at the same high pressure. The FM image exhibits a high surface concentration of solid domains with diameter $\sim 30 \mu \mathrm{m}$. The distance between the edges of adjacent solid patches is $\sim 5 \mu \mathrm{m}$, i.e. too small to be resolved by the present SPM setup, with a SPR propagation length of $\sim 10 \mu \mathrm{m}$. On the other hand, the FM is not able to discriminate between structures within the solid domains or between solid domains with different properties. We therefore propose that both domains I and II are solid but with different hydrocarbon tail tilt angles and/or refractive indices. Apart from the fact that this makes the FM image consistent with that obtained from the SPM, several other experimental findings are in line with this interpretation:

(1) The total length of a DMPE molecule with its hydrocarbon chains in their all-trans configuration is,

(13) Johnson, P. B.; Christy, R. W. Phys. Rev. 1972, 6, 4370.

(14) Ducharme, D.; Max, J.; Salesse, C.; Leblanc, R. M. J.Phys. Chem. 1990, 94, 1925. 
depending on the orientation of the polar headgroup, in the range $2.8-3.3 \mathrm{~nm} .{ }^{15}$ This number is in close agreement with the layer thickness that we determined for domain II. Apparently, this domain corresponds to lipid molecules oriented perpendicular to the substrate surface.

(2) The layer thickness, found for I is compatible with an aliphatic chain tilt of $30-40^{\circ}$. The uncertainty in this tilt angle is mainly due to lack of knowledge about the orientation of the headgroup. (Note that the hydrophilic $\mathrm{SiO}_{2}$ surface causes the polar group to bind to the surface.). The value of the tilt angle is comparable to that found for a similar lipid (dipalmitoylphosphatidylcholine), using fluorescence polarization ${ }^{16}$ and $\mathrm{X}$-ray ${ }^{15,17}$ data.

(3) In the fluid-solid coexistence region (e.g. at $A \sim 0.6$ $\mathrm{nm}^{2} /$ molecule) the thickness of solid domains has been previously determined to be $t=2.2 \mathrm{~nm}$ for dimyristoylphosphatidic acid. ${ }^{18}$ This is in close agreement with our value for domain $I$, which was prepared at a much higher lateral pressure with an average molecular area of $\sim 0.4 \mathrm{~nm}^{2} /$ molecule. Apparently, at a lateral pressure $>30$ $\mathrm{mN} / \mathrm{m}$, a new two-solid-phase equilibrium exists within DMPE monolayers. A very similar situation has been recently described by Grayer Wolf et al. ${ }^{19}$ for a surfactant monolayer of an amino acid molecule. Synchrotron X-ray experiments enabled them to follow the phase equilibrium as a function of lateral pressure. It was concluded that two solid phases coexisted with tilt angles of $16^{\circ}$ and $26^{\circ}$, respectively.

\section{Conclusions}

Evidence has been presented that SPM, capable of resolving vertical structures $<1 \mathrm{~nm}$, is able to reveal details in lipid domain structures that are not available by FM: At high lateral pressure solid lipid domains were found having different SPR optical characteristics. Our deter-

(15) Helm, C. A.; Möhwald, H.; Kjaer, K.; Als-Nielsen, J. Europhys. Lett. 1987, 6, 697 .

(16) Moy, V. T.; Keller, D.; Gaub, H. E.; McConnell, H. M. J. Phys. Chem. 1986, 90, 3198. 4575 .

(17) Janiak, M. J.; Small, D. M.; Shipley, G. G. Biochemistry 1976, 15,

(18) Helm, C. Thesis, Technische Universität, München, 1988.

(19) Grayer Wolf, S.; Deutsch, M.; Landau, E. M.; Lahav, M.; Leiserowitz, L.; Kjaer, K.; Als-Nielsen, J. Science 1988, 242, 1286. mination of the tilt angle in domain I was necessarily a rough estimate; additional information concerning the orientation of the headgroup is necessary. A possible way seems to perform laterally resolved surface-potential experiments, ${ }^{20}$ as it can be expected that this parameter is very sensitive to the headgroup orientation.

It is clear that more SPM experiments are needed to further substantiate these findings and to further characterize the solid domain structure. In particular, experiments with the lateral pressure as a parameter could be informative.

Also from an optical point of view, SPM as a method needs refinement. Apart from obvious improvements, such as a better lateral resolution and a more sophisticated image processing system, SPM would be much more useful if it were possible to do separate determinations of the (anisotropic) refractive index and layer thickness. This could provide valuable information on lipid chain packing. We have previously indicated which routes can be followed if birefringence is absent; ${ }^{21}$ for an adsorbed protein layer we have experimentally demonstrated the feasibility of one of those approaches. ${ }^{22}$

Acknowledgment. R.P.H.K is grateful to the Stichting voor Technische Wetenschappen (STW) for partial funding of his sabbatical stay at the University of Toronto. U.J.K acknowledges primary financial support for the work from the Natural Sciences and Engineering Research Council of Canada. The Ontario Laser and Lightwave Research Center (OLLRC) is acknowledged for loan of their equipment during the early stages of this project. Mr. D. Foster did most of the chemical work; Dr. G. Winston (Department of Electrical Engineering) prepared the $\mathrm{SiO}_{2}$ layers. Reno Debono, John Brennan, and Steve Brown are acknowledged for informative discussions concerning lipid chemistry and the art of handling the Langmuir-Blodgett trough.

(20) Heckl, W. M.; Baumgärtner, H.; Möhwald, H. Thin Solid Films $1989,173,269$.

(21) Kooyman, R. P. H.; de Bruijn, H. E.; Eenink, R G.; Greve, J. J. Mol. Struct. 1990, 218, 345.

(22) de Bruijn, H. E.; Kooyman, R. P. H.; Greve, J. Opt. Commun. $1991,82,425$. 